\title{
Ein Wettbewerb mit zwei Siegern
}

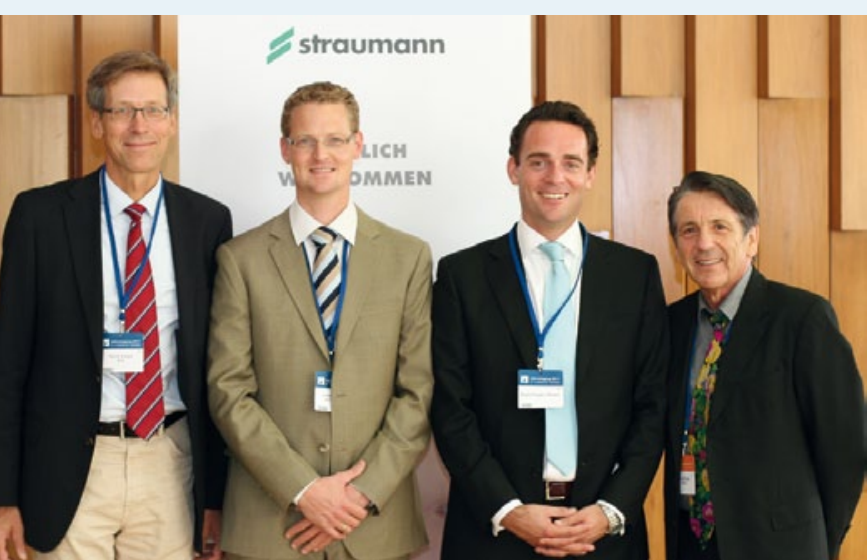

Die Preisträger mit der Jury (v.l.n.r.): Prof. Søren Jepsen, Uniklinik Bonn; Dr. Jochen Tunkel, Bad Oeynhausen; Dr. Daniel EnglerHamm, München; Dr. Bernd Heinz, Hamburg.

Die diesjährige Jahresversammlung der Deutschen Gesellschaft für Parodontologie (DGP) im September in Baden-Baden nutzte Straumann im Rahmen eines eigenen Symposiums als Plattform für die Präsentation ihrer besten Wettbewerbsteilnehmer. Das diesjährige Tagungsthema zum Spannungsfeld zwischen Lifestyle und Lebensqualität „Gesunde Zähne für ein schöneres und besseres Leben ohne kurzlebigen Lifestyle-Trends aufzusitzen" war für Straumann der Anstoß, das Leistungsvermögen seines regenerierenden Materials Emdogain als Therapie zur Behandlung von parodontalen Knochendefekten in den Fokus zu bringen. Im Vorfeld der Tagung hatten sich interessierte Zahnärzte mit ihrem besten Fall bei dem Unternehmen beworben. Man suchte Behandler, die ihren persönlichen Patientenfall mit dem Schmelzmatrixprotein Emdogain zur Beurteilung stellen wollten. Aufgabe war, seine Erfahrungen in der Rezessionsdeckung mit dem regenerierenden Material zu dokumentieren. Aus zahlreichen Fallbeispielen entschied sich die Jury final für 2 Zahnmediziner, die im Rahmen der Jahresversammlung der DGP ihre Fälle präsentieren konnten. Der 1. Platz ging an Dr. Jochen Tunkel, Bad Oeynhausen (Deckung parodontaler Rezessionen mit Straumann Emdogain und einem Bindegewebstransplantat im Splitmouth-Design) und der 2. Platz an Dr. Daniel Engler-Hamm, München (Deckung parodontaler Rezessionen mit Tunneltechnik im Oberkiefer).

Nach einer Pressemitteilung der Straumann GmbH, Freiburg Internet: www.straumann.de

\section{Chairside-Zahnaufhellungs-System mit erhöhtem Wirkstoffgehalt}

Das Chairside-Zahnaufhellungs-System Opalescence Boost hat seit der IDS einen erhöhten Wirkstoffgehalt von $40 \% \mathrm{H}_{2} \mathrm{O}_{2}$.

\section{$\ldots$ BOOST}
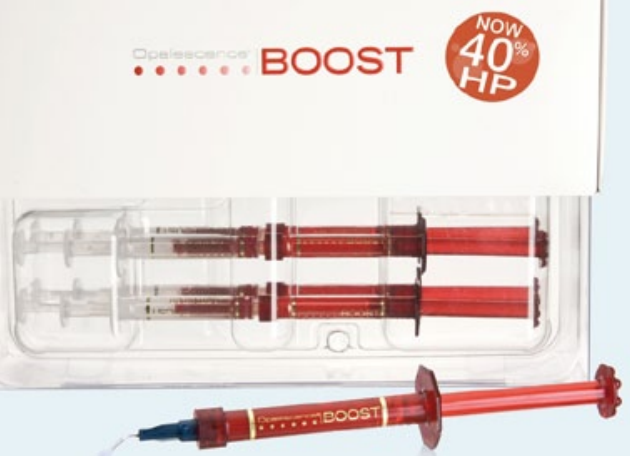

Nun haben die Tester des bekannten „Clinicians Report“ das Material unter die Lupe genommen. Sie bescheinigen ihm bereits nach $2 \times 20$ min Anwendung in einer Sitzung eine hohe Effizienz, wobei Lichtapplikation nicht nötig ist. Als Vorteile hervorgehoben werden auch die Spritzenapplikation, die Konsistenz des Gels sowie nur sehr selten auftretende Zahnsensibilitäten. Insgesamt halten 95\% das Material für „exzellent“ oder „gut“ und empfehlen ihren Zahnarzt-Kollegen den Test in den eigenen Praxen.

Nach einer Pressemitteilung der

Ultradent Products, Köln

Internet: www.updental.de

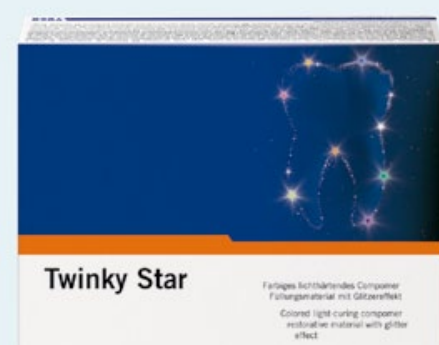

\section{บแอ}

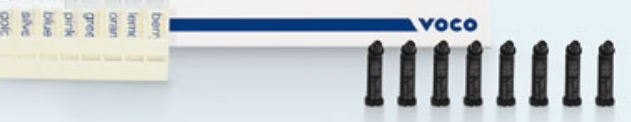

Farbiges Füllungsmaterial speziell für Milchzahnrestaurationen

\section{Für eine gute Compliance}

Twinky Star von VOCO ist ein farbiges, eigens für Kinder entwickeltes Füllungsmaterial, das sich vielfach in der Praxis bewährt hat. Aufgrund seiner einzigartigen Ausstattung und hohen Akzeptanz bei Kindern, seiner soliden Materialeigenschaften und seines einfachen Handlings wird das Produkt in aktuellen Fallstudien und Fortbildungen zur Milchzahnrestauration ausdrücklich empfohlen. Das Füllungsmaterial weckt mit seinen Effektfarben (Gold, Silber, Blau, Pink, Grün, Orange, Lemon und Brombeere) das Interesse der kleinen Patienten und erhöht deren Kooperationsbereitschaft während der Behandlung. Darüber hinaus sind die bunten Füllungen eine wirksame Motivationshilfe für die häusliche Mundhygiene. Das Material basiert auf der bewährten Kompomer-Technologie. Es überzeugt mit physikalischen Eigenschaften, wie einem hohen Füllstoffgehalt (77,8Gew.\%), einer geringen Polymerisationsschrumpfung (2,0Vol.\%), einer guten Adhäsion und Randadaptation (Schmelz-/Dentinhaftung von 27,3 bzw. 24,1 MPa), einer hohen Druckhärte (316 MPa), einer ausgeprägten Biegefestigkeit (116 MPa), einer geringen Abrasion $(15 \mu \mathrm{m})$ und einer hohen Biokompatibilität. Mit seiner ergänzenden Fluoridabgabe trägt es zur Vorbeugung gegen Sekundärkaries bei. Mit seiner Stabilität und Farbbeständigkeit halten die mit Twinky Star gelegten Füllungen sicher und dauerhaft bis zur Exfoliation. Das Material lässt sich aufgrund seiner guten Konsistenz bequem stopfen und modellieren und ist leicht zu polieren.

Nach einer Pressemitteilung der voco GmbH, Cuxhaven

Internet: www.voco.de 


\section{Mehr Gesamtlånge für den Einsatz bei tiefen Kavitåten}

Die vielfältigen Instrumente aus Hochleistungskeramik haben ihren festen Platz im Markt gefunden. Durch sie sind Ästhetik, Bioverträglichkeit und Feingefühl in die Praxen eingezogen. Der neueste Zuwachs ist der Fissurenbohrer K59: Er wurde in Zusammenarbeit mit Dr. Ahmed El Hoshy, Universität Kairo/Ägypten, entwickelt und ist durch sein filigranes Arbeitsteil für das minimal-invasive Aufziehen von Fissuren geeignet. Seine Indikation ist das leichte Aufziehen von Fissuren. Dies kann im Rahmen einer erweiterten Fissurenversiegelung oder der Kariesdetektion erforderlich sein.

Neuigkeiten gibt es auch zum CeraBur K1SM. Den beliebten weißen Bohrer für

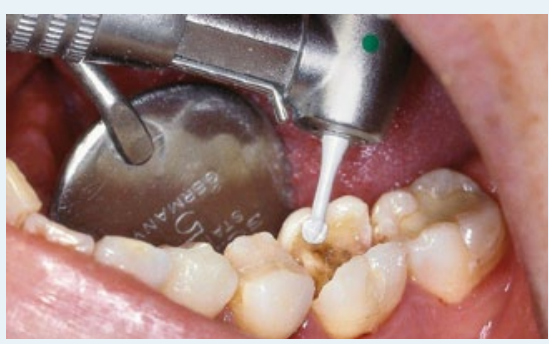

minimales, taktiles Exkavieren gibt es nun von Größe 010-023 mit 4 mm mehr Gesamtlänge (205er Schaft). Dies ermöglicht den Einsatz bei tieferen Kavitäten und kommt dem Anwender bei der Präparation einer Zugangskavität für eine nachfolgende endodontische Behandlung zugute.

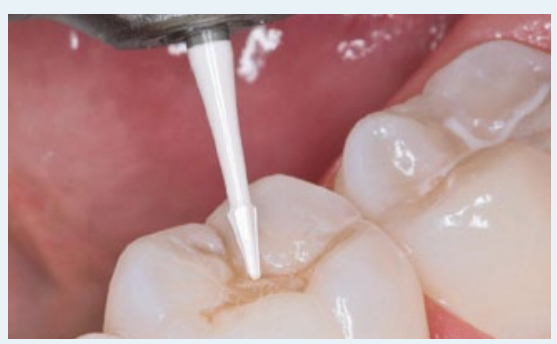

Mehr Details rund um die Keramikfamilie sind in der CeraLine-Broschüre (402435V5) zu finden, die unter www. kometdental.de schnell und unkompliziert angefordert werden kann.

Nach einer Pressemitteilung der

Komet Gebr. Brasseler GmbH \&Co KG, Lemgo Internet: www.kometdental.de

\section{Für Bulk-Fülltechnik geeignet}

„SonicFill zeigte bei Applikation in einer einzigen, bis zu $5 \mathrm{~mm}$ dicken Schicht die gleiche Randdichtigkeit im Dentin und Schmelz wie andere derzeit erhältliche Bulk-Fill-Komposite oder konventionell geschichtete Komposite“, so Prof. Roland Frankenberger zum Ergebnis seiner Komposit-Vergleichsstudie [1]. Es braucht keine Überschichtung, wie eine Untersuchung von Jeffrey et al. [2] zeigte. Laut den US-Wissenschaftlern ist „die Druck- festigkeit höher als bei den meisten in dieser Studie getesteten Füllungskompositen. SonicFill ermöglicht somit dauerhafte, langlebige Füllungen, die der hohen Kaubelastung im Seitenzahnbereich standhalten“. Die Studiensammlung steht ab sofort unter www.SonicFill.eu oder www.kavo.com/sonicfill-studien zum Download bereit.

Derzeit besteht für Interessenten die Möglichkeit, das System selbst zu testen.
Weitere Informationen hierzu finden sich unter www.kavo.com/sonicfill.

Literatur

1 Randqualität und assoziierte Höckerdeformation bei SonicFill TM Füllungen. Prof. Dr. Roland Frankenberger, Philipps-Universität Marburg, Deutschland, 2011

2 Dr. Jeffrey Y. Thompson, NOVA Southeastern University, Ft. Lauderdale, FL, USA. Eingereicht bei der AADR 2010

Nach einer Pressemitteilung der

Kerr GmbH, Rastatt

Internet: www.kerrdental.de

\section{Die „State-of-the-art"-Lösung bei heiklen Endo-Problemen}

Zur Behandlung schwieriger endodontischer Fälle bietet DENTSPLY Maillefer das ProRoot MTA (Mineral Trioxid Aggregat) an, das sich zur Pulpa-Überkappung und zur Reparatur von Wurzelresorptionen seit Jahren bewährt. Jetzt können Zahnärzte zu dessen Applikation auch das MAP-System (MAP= Micro-Apical Placement) nutzen. Es enthält sämtliche benötigten mikrochirurgischen Komponenten, einschließlich biegbarer Spitzen mit Memory-Effekt. Der achsengerechte Zugang zur Kavität wird durch die Geometrie der Instrumente wesentlich vereinfacht. Beispielsweise kann damit eine retrograde Obturation beginnend vom
Fundus der Kavität suffizient durchgeführt werden, wobei Einschlüsse oder Versprengungen des Füllmaterials leichter vermieden werden können. Dieses mikrochirurgische Instrumentarium ermöglicht die exakte und zeitsparende Platzierung von ProRoot MTA im Apex des Wurzelkanals.

Weitere Informationen zum MAP-System sowie zu ProRoot MTA sind unter der gebührenfreien DENTSPLY-Service-Line für Deutschland 08000/735000 sowie im Internet unter www.dentsply.de abrufbar. Details zum Endodontie-Kongress können eingesehen werden unter www.endocongress-maillefer.de.

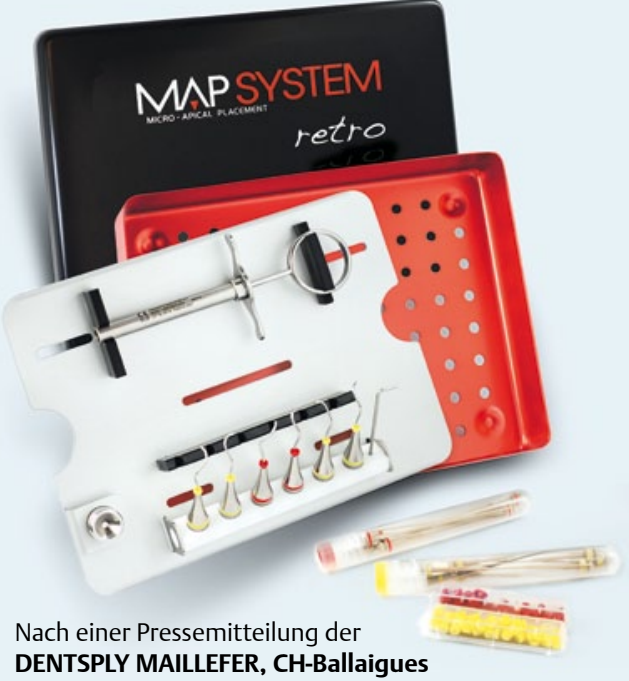




\section{Nachhaltige Implantologie}

\section{fokussiert Periimplantitis}

Vor 5 Jahren gründete sich auf Initiative des Schweizer Implantatherstellers Clinical House die Europe die Academy of Periointegration als Zusammenschluss von internationalen Implantologen, Parodontologen und Implantatentwicklern. Im Februar 2007 definierte die Academy of Periointegration auf Basis wissenschaftlicher Erkenntnisse 4 Designkriterien für periointegrative dentale Implantate. Clinical House Europe hat diese festgelegten Designkriterien in dem periointegrativen Implantatdesign von PerioType X-Pert umgesetzt. Die Zirco-Seal $^{{ }_{-}}$ Oberfläche des Abutments ist mit Zirkonnitrid beschichtet. Dieses vom Fraunhofer-Institut Braunschweig und der Universität Düsseldorf entwickelte Material ist 6-mal härter als Reintitan und verringert ein Anhaften des Biofilms mit pa-

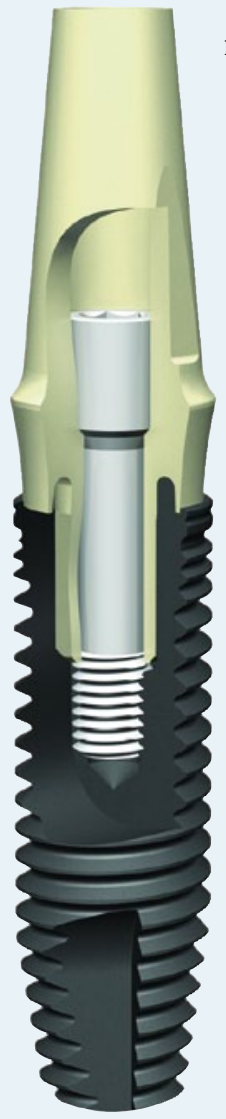

radontalpathogenen Keimen erheblich. Die Anlagerung der Gingiva zu einem dicht anliegenden Saumepithel wird gefördert. Die stegförmige Implantatschulter mit Platform-Switch verhindert das Eindringen von Bakterien ins Innere des Implantats und gewährleistet in Kombination mit der stabilen oktagonalen Innenverbindung eine optimale Kraftübertragung mit reduzierten Mikrobewegungen. Die Perio-Coat ${ }^{\circledR}$-ImplantatOberfläche wird mit anerkannter wissenschaftlicher Dokumentation seit über 20 Jahren in der Implantologie eingesetzt. Das PerioType-XPert-Implantat wurde in der Schweiz entwickelt und in Deutschland produziert.

Nach einer Pressemitteilung der Clinical House Dental GmbH, Bochum

Internet: www.periotype.de

\section{Präziser Abdruck mit Langzeiterfolg}

Um eine exakte Wiedergabe bei der Abformung zu erreichen, ist eine Retraktion der Gingiva erforderlich. Sie erfolgt mithilfe von Retraktionsfäden- oder ringen, die vor der Abdrucknahme mit Flüssigkeiten getränkt werden können. Hierbei hat sich Aluminiumsulfat, wie in ORBAT von lege ar-

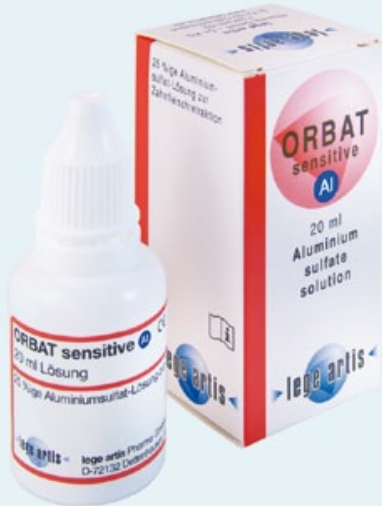

rüber hinaus enthält die Lösung keinerlei gefäßverengende Zusätze und kann somit auch bei Patienten mit kardiovaskulären Erkrankungen angewandt werden. Zur Zahnfleischretraktion wird der Faden vor Abdrucknahme 1-3 min in der Lösung getränkt. Anschließend wird der Retraktionsfaden entfernt und bei Betis sensitive enthalten, bewährt, da es adstringierend ist und damit eine lokale Blutstillung bewirkt. Ein aktueller Test hat ergeben, dass ORBAT sensitive keine Beeinträchtigungen auf herkömmliche Abformmaterialien hat. Die Aluminiumsulfat-Lösung weist eine sehr gute Verträglichkeit mit den am häufigsten verwendeten Abformmaterialien auf A-Silikon-Basis und Polyäther auf. Da- darf gespült. Bei Exsudation und Zahnfleischbluten kann die 25\%ige Aluminiumsulfat-Lösung nach dem Abtupfen mit einem getränkten Wattepellet unter leichtem Druck direkt aufgetragen werden.

Nach einer Pressemitteilung der

lege artis Pharma $\mathbf{G m b H}+\mathbf{C o}$. KG, Dettenhausen

Internet: www.legeartis.de
Vertriebskooperation

\section{Ideale Membran für jede Indikation}

Ab Mitte November sind die Cytoplast ${ }^{\circledR}$ d-PTFE-Membranen, Cytoplast ${ }^{\circledR}$-Kollagenmembranen und das Cytoplast ${ }^{\circledR}$-PTFENahtmaterial bei RIEMSER Dental erhältlich. Die beiden Unternehmen RIEMSER Arzneimittel AG und Osteogenics Biomedical, Inc., USA, haben eine Vertriebskooperation für Deutschland, Österreich und Frankreich geschlossen. Mit der Erweiterung des Portfolios um die Cytoplast ${ }^{\circledR}$-Barrieremembranen wird RIEMSER Dental jetzt das umfangreichste Membranportfolio anbieten, das es in diesen Ländern gibt und so in der Lage sein, die ideale Membran für jede Indikation zu liefern.

Dr. Michael Leible, Leiter des Geschäftsbereichs Dental der RIEMSER Arzneimittel AG betont den Kundennutzen: „Die aktuelle Sortimentserweiterung belegt, dass wir unsere Zielsetzung im Geschäftsbereich Dental konsequent leben, nämlich den Kunden Comprehensive Oral Surgery Technologies zu bieten. Die Cytoplast-Produkte ersetzen zum einen die nicht mehr erhältliche TefGenTM*-Membran, zum anderen bieten sie mit den titanverstärkten Modifikationen raumschaffende, nicht resorbierbare Barrieren."

Mittlerweile stehen 8 Konfigurationen titanverstärkter Membranen zur Raumhaltung bzw. Abschirmung von Knochendefekten aller Art zur Verfügung stehen.

Nach einer Pressemitteilung der

RIEMSER Arzneimittel AG, Kleinostheim

Internet: www.RIEMSER-Dental.com 